\title{
Rendimiento académico y evaluación de egreso en un Programa de Estomatología
}

\author{
Academic performance and graduation evaluation in a Program of Stomatology \\ Mariana Cutipa Luque 1,a, Verónica Chávez Lovón ${ }^{1, a}$, Milagros Carrasco Loyola ${ }^{2, b}$.

\section{RESUMEN}

Objetivo: Determinar la relación entre el rendimiento académico y la evaluación de egreso en estudiantes de estomatología. Material y métodos: El estudio fue observacional, descriptivo y transversal. La población estuvo constituida por el registro de 45 egresados. Las variables de estudio fueron rendimiento académico en los internados y el rendimiento en la evaluación final de carrera. Resultados: El Examen de Suficiencia Profesional (ESP) promedio fue 11,82 (Mín=8; Máx=15,20) y el Rendimiento académico promedio fue 15,87 (Mín=9,03; Máx=18,40), encontrando una correlación positiva baja (Spearman=0,205). El rendimiento académico promedio en las asignaturas del último año fue en el área de clínica en pacientes adultos, 14,92; clínica en pacientes pediátricos, 15,76; en el área hospitalaria, 15,02; y en el área de salud pública estomatológica, 17,80. El ESP promedio fue: en el área de clínica en pacientes adultos, 11,10; cínica en pacientes pediátricos, 13,83; área hospitalaria, 10,31; salud pública estomatológica, 12,02. Se encontró una correlación mínima a baja en las áreas clínicas y social; en el área hospitalaria la correlación fue negativa mínima. Conclusiones: Existe una tendencia a mejores puntajes en el ESP y el Rendimiento académico en las estudiantes mujeres en comparación a los hombres. No hay relación entre los resultados del Examen de Suficiencia Profesional y el rendimiento académico de las asignaturas e internados del Programa de Estomatología de la Universidad Peruana Cayetano Heredia.

PALABRAS CLAVE: Evaluación educacional, rendimiento académico, carrera profesional.

\section{ABSTRACT}

Objective: To determine the relationship between academic performance and the exit evaluation in stomatology students. Material and Methods: The study was observational, descriptive, and cross-sectional. The population consisted of the registry of 45 graduates. The study variables were performance in the final evaluation of the degree (Professional Proficiency Exam) and academic performance in the final year. Results: The average Professional Proficiency Exam (PPE) was $11.82(\mathrm{Min}=8$; $\mathrm{Max}=15.20)$ and the average academic performance was $15.87(\mathrm{Min}=9.03$; Max $=18.40)$, finding a correlation low positive (Spearman=0.205). The average of the academic performance in the subjects in the clinical area in adult patients 14.92; clinic in pediatric patients 
15.76; in the hospital area 15.02; and in the areas of oral public health 17.80. The average of the Professional Proficiency Exam was: in the clinical area in adult patients 11.10; clinic in pediatric patients 13.83; hospital 10.31; social dentistry 12.02. A minimum to low positive correlation was found in the clinical and social area; in the hospital area the correlation was minimal negative. Conclusion: There is a trend for better scores on PPE and academic performance in female students compared to males. There is no relationship between the results of the PPE and the academic performance of the internship and subjects of the last year of the Stomatology Program of the Peruvian University Cayetano Heredia.

KEYWORDS: Educational evaluation, academic performance, Professional Competence.

\section{INTRODUCCIÓN}

Una educación universitaria de calidad exige indicadores de eficiencia académica. El modelo de acreditación de la calidad educativa para los programas de estudios de nuestro país enfatiza en la evaluación del logro de las competencias durante el proceso formativo y de las competencias del perfil de egreso. La evaluación de los estudiantes tiene como una de sus funciones la mejora del aprendizaje (1).

En muchos países se aplican pruebas estandarizadas al finalizar la carrera para obtener la titulación. E1 Examen General para el Egreso de la Licenciatura (EGEL) en México, evalúa conocimientos y habilidades necesarios para poder ejercer la profesión y es implementado por el Centro Nacional para la Evaluación de la Educación Superior $(2,3)$. En Argentina, el Análisis de Contenidos y Competencias que Efectivamente Disponen los Estudiantes (ACCEDE), se aplica a estudiantes que estén por graduarse con el propósito de recolectar información cualitativa sobre las competencias incorporadas (4). En Brasil, a través de un examen anual a los estudiantes en condiciones de concluir sus estudios y de carácter obligatorio, se retroalimenta los procesos en la toma de decisiones para mejorar de la educación de los estudiantes próximos a graduarse $(5,6)$. En los Estados Unidos, la Comisión Conjunta de Exámenes Dentales Nacionales (National Board Dental Examination - NBDE) aplica un examen que evalúan la capacidad de los candidatos $(7,8,9)$.

En el Perú, en el 2003 se realizó un piloto del Examen Nacional de Medicina (ENAM), a cargo de la Asociación Peruana de Facultades de Medicina (ASPEFAM). Años después, se consolidó el proceso del ENAM y se hacen públicos los resultados a partir del 2008. Actualmente, es requisito para la obtención de la colegiatura, postulación a la residencia y la elección de las plazas donde los egresados realizan el Servicio Rural Urbano Marginal en Salud (SERUMS). Además, sirve como instrumento de evaluación de la calidad de la educación médica durante la formación de pregrado, promoviendo la mejora de la educación médica y la revisión crítica del perfil de formación $(10,11,12)$.

Recientemente, se aplica el Examen Nacional de Odontología (ENAO), implementado por la Asociación Peruana de Facultades de Odontología, como un instrumento que permite medir la calidad educativa de los odontólogos del país, además establece por orden de mérito la distribución de plazas para participar en el SERUMS. Corresponde un valor del $70 \%$ al examen y $30 \%$ al promedio ponderado general de estudios de pregrado sin incluir internado para el cálculo final del puntaje $(13,14)$.

La Facultad de Estomatología de la Universidad Peruana Cayetano Heredia (UPCH), desde el año 2015 viene aplicando el Examen de Suficiencia Profesional (ESP), a los estudiantes del último año en relación a las áreas de Clínica Estomatológica Integral del Adulto, Clínica Estomatológica Integral Pediátrica, Hospitalario Estomatológico y Odontología Social o Salud Pública Estomatológica, con la finalidad de evaluar los conocimientos adquiridos por los estudiantes durante su formación profesional.

El ESP consta de una prueba escrita de 100 preguntas objetivas de conocimientos, divididas en cuatro secciones: clínica del adulto, clínica pediátrica, hospitalario y odontología social. Se aplica al término de los cinco años de estudios durante 120 minutos. Los resultados son procesados por la Dirección de la Escuela Profesional y reportados al Vicedecanato 
para las acciones pertinentes. El examen consta principalmente de preguntas objetivas de opción múltiple y verdadero / falso. Además, se plantean cuatro casos clínicos, una por cada área odontológica $\mathrm{y}$ algunas preguntas abiertas.

Por otro lado, en el programa de Estomatología de la UPCH, se integran y consolidan los conocimientos en el último año de estudios mediante las asignaturas de Clínica Estomatológica Integral del Adulto y Clínica Estomatológica Integral Pediátrica, así como el Internado Hospitalario Estomatológico e Internado de Odontología Social, constituyendo un periodo propicio de evaluación del estudiante. Son tres rotaciones de cuatro meses cada una, constituyen un importante escenario de prácticas en la enseñanza superior de la carrera de Estomatología, mereciendo el interés de educadores en odontología y otras ciencias de la salud en el país y en la región, desde los inicios de su creación (15).

En las asignaturas de Clínica Estomatológica Integral del Adulto y Pediátrica, los estudiantes realizan tratamientos estomatológicos integrales diversos abarcando las diferentes especialidades que la estomatología posee. En la presentación del caso clínico de cada paciente al docente supervisor, sustentan el diagnóstico $\mathrm{y}$ definen el plan de tratamiento.

El Internado Hospitalario Estomatológico, consta de rotaciones por diversas especialidades médicas y realizan guardias diurnas y nocturnas en el área de emergencia del Hospital Nacional Cayetano Heredia. Los conocimientos de Medicina Oral, Cirugía Oral, Patología Oral y Farmacología necesitan ser aplicados en esta asignatura.

En el Internado de Odontología Social, se realiza un abordaje poblacional de la salud bucal. El estudiante realiza el diagnóstico epidemiológico de salud bucal de la comunidad rural o urbano marginal a la cual es asignado. Posteriormente, planifica, ejecuta y evalúa su intervención. Además, se inserta en un servicio de salud y participa con el equipo de salud en actividades de prevención y promoción. Esta experiencia permite al estudiante vivir la realidad del país, con una mayor perspectiva para la mejora de la salud de la población (16).
El objetivo del estudio fue determinar la relación entre los resultados del ESP y el rendimiento académico de las asignaturas clínicas y los internados del Programa de Estomatología de la Universidad Peruana Cayetano Heredia, Lima- Perú 2019.

\section{MATERIAL Y MÉTODOS}

El estudio fue observacional, descriptivo y transversal. La población de estudio fueron los estudiantes de la Facultad de Estomatología de la UPCH que egresaron en el año 2018. Se seleccionó a todos los que cumplieron los criterios de inclusión: haber rendido el ESP y con registros completos, la cual estuvo constituida por 45 egresados.

Las variables del estudio fueron rendimiento académico en las asignaturas del último año de estudios del programa de estudios y el resultado del ESP. El rendimiento académico en el quinto año de estudios, consideró las calificaciones obtenidas en las asignaturas de Clínica Estomatológica Integral del Adulto, Clínica Estomatológica Integral Pediátrica, Internado Hospitalario Estomatológico e Internado de Odontología Social. El resultado obtenido en el ESP se desagregó en puntajes alcanzados en las preguntas correspondientes cada área: clínica del adulto, clínica pediátrica, hospitalario y odontología social (salud pública).

Los datos de los estudiantes y los resultados del ESP fueron solicitados a la Dirección de la Carrera de Estomatología de la Facultad de Estomatología de la UPCH. Posteriormente, se generó una base de datos con la información codificada por las investigadoras para conservar el anonimato de los estudiantes y se convierten a escala vigesimal los resultados del ESP. Se realizó un análisis descriptivo mediante la obtención de las frecuencias absolutas y relativas de las variables cualitativas, para las variables cuantitativas, se obtuvieron promedios y desviación estándar. Se evaluó su normalidad con la prueba de Shapiro Wilks, para la correlación se empleó la prueba de Spearman. En el estudio se empleó el programa Microsoft Excel y el programa estadístico SPSS 24.0; con un nivel de confianza del $95 \%$ y un $\mathrm{p}<0.05$.

Este estudio fue aprobado el Comité Institucional de Ética en Investigación de la Universidad Peruana 
Cayetano Heredia (CIEI-UPCH), el 11.02.2019, con el código SIDISI 103774.

\section{RESULTADOS}

El estudio se realizó en 45 estudiantes de la Facultad de Estomatología de la UPCH, donde 33 $(73,33 \%)$ fueron mujeres y 12 hombres $(26,67 \%)$. Los resultados del ESP y el rendimiento académico del último año de estudios están expresados en escala vigesimal.

El 77,78\% de los estudiantes aprobaron el ESP. Solo uno (2.22\%) desaprobó alguna de las asignaturas del último año de estudios. El Rendimiento académico y el ESP por cada estudiante se ilustran en el Gráfico 1.
El ESP promedio fue 11,82 siendo la nota mínima de 8 y la máxima 15,20. El rendimiento académico promedio fue 15,87 siendo la nota mínima de 9,03 y la máxima de 18,40 . Se encontró una correlación positiva baja $(0,205)$ entre los promedios del ESP y Rendimiento académico (tabla 1 ).

Las mujeres lograron un mayor puntaje en el ESP en las áreas de clínica del adulto y pediátrica y en odontología social:11,52, 13,96 y 12,29, respectivamente, en comparación a los varones: $9,97,13,47$ y 11,27). Similarmente, en el último año de estudios, las mujeres lograron un mayor Rendimiento académico en las asignaturas de Clínica Estomatológica Integral del Adulto, Clínica Estomatológica Integral Pediátrica y los internados Hospitalario Estomatológico y Odontología Social:

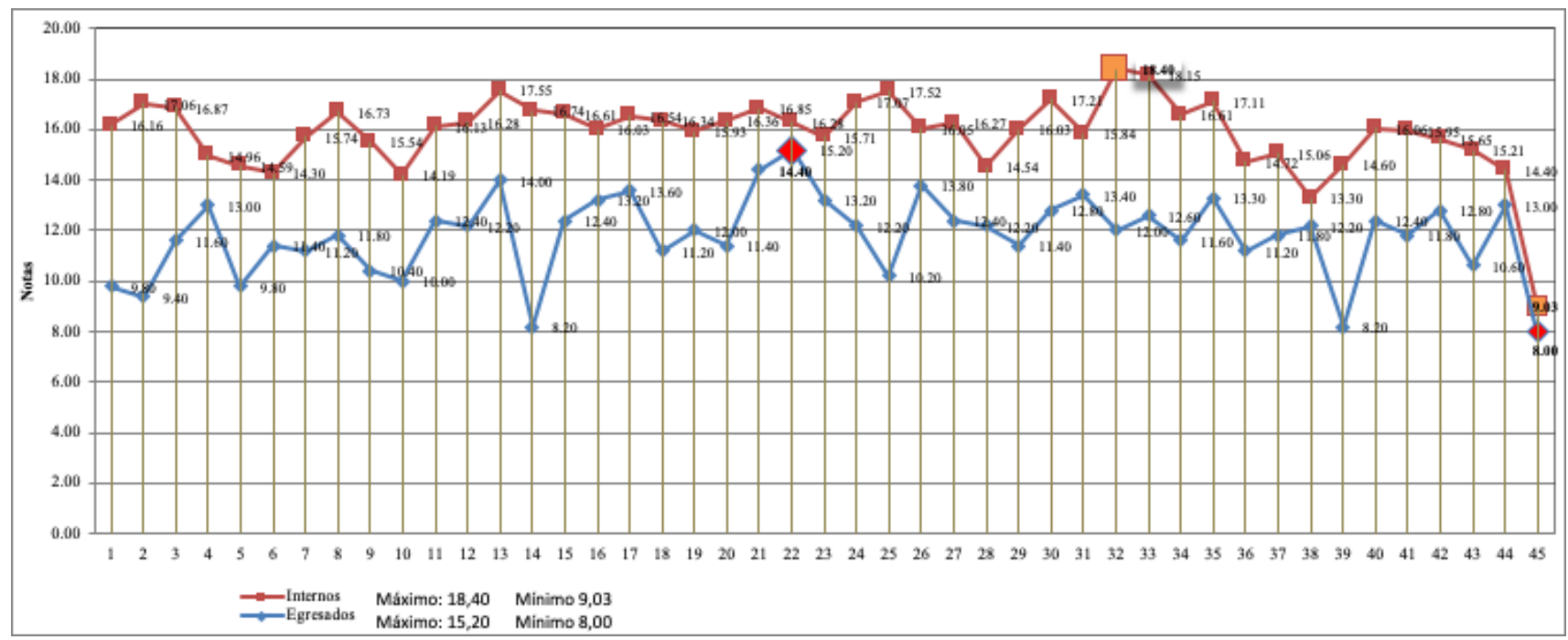

Gráfico 1. ESP y rendimiento académico en los internados, según egresado

Tabla 1. Resultados del ESP y Rendimiento académico.

\begin{tabular}{lcccc}
\hline \multicolumn{1}{c}{ Variables } & Promedio & D.E. & Mínimo & Máximo \\
\hline ESP & & & & \\
CA & 11,10 & 2,44 & 0,00 & 16,00 \\
CP & 13,83 & 2,07 & 8,00 & 16,80 \\
HE & 10,31 & 2,21 & 3,20 & 15,20 \\
OS & 12,02 & 2,32 & 5,60 & 16,00 \\
Rendimiento académico & & & & \\
CA & 14,92 & 2,85 & 5,01 & 18,27 \\
CP & 15,76 & 2,72 & 2,75 & 18,41 \\
HE & 15,02 & 0,90 & 13,15 & 17,32 \\
OS & 17,80 & 1,62 & 13,20 & 20,00 \\
\hline
\end{tabular}

CA: Clínica Estomatológica Integral del Adulto. HE: Hospitalario Estomatológico.

CP: Clínica Estomatológica Integral Pediátrica OS: Odontologia Social.

$\mathrm{DE}=$ Desviación estándar 
Tabla 2. Resultados del ESP y rendimiento académico, según sexo.

\begin{tabular}{|c|c|c|c|}
\hline & Variables & Hombres & Mujeres \\
\hline \multicolumn{4}{|l|}{ ESP } \\
\hline & $\mathrm{CA}$ & 9,97 & 11,52 \\
\hline & $\mathrm{CP}$ & 13,47 & 13,96 \\
\hline & $\mathrm{HE}$ & 10,93 & 10,08 \\
\hline & OS & 11,27 & 12,29 \\
\hline
\end{tabular}

Rendimiento académico

$\begin{array}{lll}\text { CA } & 13,20 & 15,54 \\ \text { CP } & 14,36 & 16,26 \\ \text { HE } & 14,65 & 15,15 \\ \text { OS } & 17,02 & 18,08\end{array}$

CA: Clínica Estomatológica Integral del Adulto.

HE: Hospitalario Estomatológico.

CP: Clínica Estomatológica Integral Pediátrica

OS: Odontologia Social. es positiva baja en clínica del adulto y pediátrica, mientras que es positiva mínima para salud pública. En el área hospitalaria la correlación fue negativa mínima tabla 3.

\section{DISCUSIÓN}

El ESP es una evaluación de carácter multidisciplinaria aplicada a profesionales para demostrar que tienen las capacidades para el ejercicio profesional (17). El programa de Estomatología implemento el ESP en estudiantes del último año de estudios, en cumplimiento a los estándares para la acreditación de carreras de odontología, actualmente no vigentes, propuesto por el Sistema Nacional de Evaluación, Acreditación y Certificación de la Calidad Educativa (SINEACE), que hacía mención a una evaluación de conocimientos al final de la carrera

Tabla 3. Resultados del ESP y rendimiento académico.

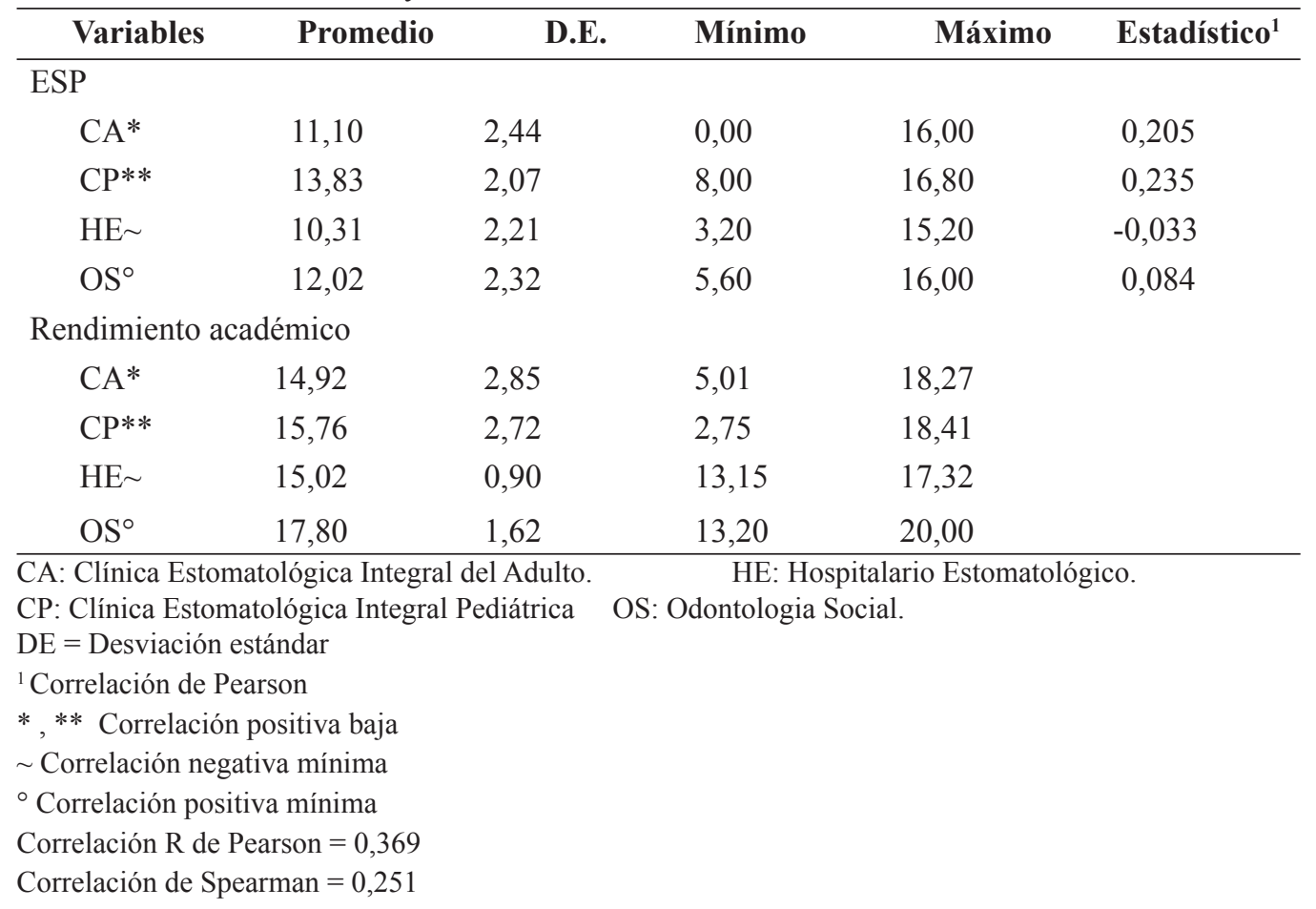

$15,54,16,26,15,15$ y 18,08 , respectivamente, en comparación a los hombres:13,20,14,36, 14,65 y 17,02 (tabla 2).

Se encontró una correlación positiva baja entre los promedios de ESP y Rendimiento académico: 0,205. Cuando se evaluó la correlación entre las áreas del ESP y del Rendimiento académico, se encuentra que profesional, cuyos resultados sean utilizados para la mejora del proyecto educativo (18).

Al analizar los resultados de este estudio, no se encontró correlación estadísticamente significativa entre los resultados del ESP y el rendimiento académico de las asignaturas e internado del Programa de Estomatología de la UPCH. La ESP es 
una prueba de conocimientos y de aplicación a través de casos; sin embargo, la evaluación en las asignaturas e internados considera además la evaluación de competencias y el componente actitudinal, tal como lo plantea el plan curricular del programa de estudios para las evidencias de aprendizaje: saber, hacer, de actitud y de producto (19).

Además, el ESP promedio fue menor que el rendimiento académico en las asignaturas clínicas y los internados hospitalario y de salud pública. Un mayor promedio durante las asignaturas clínicas e internados podría deberse a que éstos tienen un alto componente práctico, lo cual representa un mejor escenario para la evaluación de las competencias.

Estos resultados mostrarían la necesidad de implementar un diseño de evaluación al egreso que evalúe las competencias del perfil de egreso y durante el proceso de enseñanza, con un enfoque por competencias. La evaluación de competencias del perfil de egreso es considerado en el modelo de acreditación para programas de estudios de educación superior universitaria del SINEACE, que refiere el estándar de logro de competencias, sobre el uso de mecanismos para evaluar que los egresados cuentan con las competencias definidas en el perfil de egreso (20).

En la carrera de Estomatología, el ESP es de carácter formativo permitiendo evaluar en el egresado los aspectos conceptuales de la especialidad como resultado de los aprendizajes obtenidos durante su formación académica. Considerando que esta prueba es tomada al término de los estudios y no conduce a una calificación vinculada al récord académico, es probable que los estudiantes no le hayan dado la importancia debida y no esté reflejando el verdadero aprendizaje sobre los temas abordados en la prueba.

En general, se observó un mayor promedio para el sexo femenino comparado a los varones. Solo en el área de Hospitalario Estomatológico del ESP, los hombres tienen un mayor promedio que las mujeres. Algunos estudios indican que las personas de sexo femenino son más receptivas a las señales emocionales y desarrollan mejor las habilidades blandas, lo que podría contribuir a una mejor relación odontólogo - paciente; sin embargo, son los hombres quienes usualmente ofrecen soluciones racionales $(21,22)$. En este sentido, es más probable que las mujeres obtengan mayor éxito académico en los internados en comparación a los hombres.

Por otro lado, un aspecto a considerar es la construcción de la prueba del ESP. Las preguntas que la componen son elaboradas por los departamentos académicos de la Facultad (Clínica Estomatológica, Estomatología del Niño y Adolescente, Medicina y Cirugía Bucomaxilofacial, Odontología Social). El número de preguntas es el mismo por cada departamento. La pertinencia de las preguntas y la coherencia en la proporción del número de preguntas sobre una materia en relación con el número de créditos, no fue evaluado en este estudio. Es posible que esta situación haya influido de alguna forma en el menor puntaje obtenido en el ESP.

Un indicador de calidad educativa es el logro de las competencias del perfil de egreso en el modelo de acreditación de programas de estudios, para la cual se requiere de escenarios reales o cercanos a él y la cual se enfrentará el futuro profesional $(23,24)$. Evaluar al egresado requiere no solo de un examen compuesto por preguntas teóricas sino también evaluar el desarrollo de las competencias de egreso propuestas. En ese sentido, se puede considerar al rendimiento en las asignaturas e internados del último año de estudios como mejores referentes de evaluación de las competencias finales del estudiante, comparado al ESP. De ahí posiblemente la discrepancia entre el rendimiento del último año estudios con el ESP.

Sin embargo, una ventaja del ESP es proporcionar al estudiante próximo a egresar, un acercamiento a la experiencia de los exámenes nacionales como el ENAO. Los resultados obtenidos en el ESP, deben permitir al programa realizar mejoras en el proceso de enseñanza para así formar profesionales que impacten positivamente en la sociedad.

Una forma de mejorar el mecanismo de evaluación al final de la carrera es mediante pruebas computarizadas aproximándose a escenarios cercanos a los reales, o a través de simuladores, que permitan evaluar las competencias del perfil de egreso con las que afrontará el futuro profesional a las demandas del ejercicio profesional (24).

Las experiencias diseñadas al término del proceso de formación de la Escuela de Estomatología 
son espacios oportunos para la evaluación de las competencias de egreso. Estas asignaturas integradoras deberían permitir al estudiante movilizar sus aprendizajes para responder a tareas complejas propias de la profesión. Ello implica además una revisión del plan curricular que asegure la alineación de las asignaturas hacia el logro del perfil de egreso.

Entre las limitaciones del estudio se encuentra que el ESP, al no ser parte del sistema de evaluación, existe el riesgo que no hayan respondido con el interés esperado. Esto no permitiría contar con un dato preciso y estimar su relación con el rendimiento del estudiante del último año. Otra limitación del ESP es que no se cuenta con una matriz de evaluación que identifique las capacidades que se están evaluando, dado que queda a discreción de cada departamento académico incorporar las preguntas que considere más importantes.

Se concluye en la investigación, que los resultados de la evaluación teórica que se realiza a través del ESP no se relacionan con el rendimiento en el último año, en una promoción de estudiantes del programa de Estomatología.

Conflicto de intereses: los autores no tienen conflicto de interés con este informe.

Financiamiento: Ninguno.

Contribuciones de los autores: todos los autores contribuyeron a este manuscrito.

\section{Correspondencia:}

Mariana Cutipa

Calle 3, Mz 144 Lt 18 AA HH San Martin de Porres. Los Olivos, Lima, Perú.

Correo electrónico: mariana.cutipa.1@upch.pe

\section{REFERENCIAS BIBLIOGRÁFICAS}

1. Sistema Nacional de la Evaluación y Certificación de la Calidad Educativa. Explicación de estándares del modelo de acreditación de programas de estudios de educación superior universitaria. Lima: SINEACE; 2018.

2. López V. Análisis del Examen General de Calidad profesional para la odontología en México. Reencuentro. 1998; 23:76-82.

3. CENEVAL. Examen Ceneval de Odontología. Características del EGEL-O. Lima: CENEVAL; 2011. (Citado el 8 de junio del 2020) Disponible en: http:// www.ceneval.net/odontologia/que.html.

4. Guerin V, Pérez Roselli C, Jeppesen C. Evaluación integral de los procesos de acreditación de grado. Buenos Aires, Argentina: Comisión Nacional de Evaluación y Acreditación; 2003.

5. Fuentes J, Silva M, Llermaly S. Examen Nacional de Odontología: Revisión Bibliográfica de Instrumentos. Int J Odontostomat. 2014; 8(1): 125-131.

6. Ministerio de Educação. Prova Brasil - Apresentação. Brasilia: Ministerio de Educação;2011. (Citado el 8 de junio del 2020) Disponible en: en: http://portal.mec. gov.br/prova-brasil

7. Duong M, Cothron A, Lawson N, Doherty E. Journal of Dental Education. 2018; 82(3): 252-259.

8. Abdelkarim A, Sullivan D. Attitudes and Perceptions of U.S. Dental Student and Faculty Regarding Dental Licensure. 2015; 79 (1) 81-88.

9. Joint Commission on National Dental Examinations. Washington DC: ADA; 2019. (Citado el 8 de junio del 2020) Disponible en: https://www.ada.org/en/jcnde/ examinations

10. Gálvez B. El Examen Nacional de Medicina (ENAM) y su rol en Perú. Horiz Med. 2019; 14(3): 56-57.

11. Torres J. Los exámenes nacionales de medicina (ENAM) en el Perú. Rev perú med exp salud publica. 2008; 25(3): 316-318.

12. Murillo J. Historia de la Asociación Peruana de Facultades de Medicina. Lima: ASPEFAM; 2018. (Citado el 8 de junio del 2020) Disponible en: http:// www.aspefam.org.pe/series/serie1.pdf

13. Ministerio de Salud. Resolución Ministerial N³392016/MINSA. Lima, Perú: Ministerio de salud; 2016. (Citado el 8 de junio del 2020) Disponible en: https:// c dn.www.gob.pe/uploads/document/ file/193616/192341_RM_339-2016-MINSA. pdf20180904-20266-2c4jeq.pdf

14. Asociación Peruana de Facultades de Odontología. Examen Nacional de Odontología.Lima: Asociación Peruana de Facultades de Odontología. (Citado el 8 de junio del 2020) Disponible en: https://aspefo.org/enao

15. Beltrán R. Educación en odontología: manual del profesor. Lima: Universidad Peruana Cayetano Heredia; 1998.

16. Universidad Peruano Cayetano Heredia. Malla curricular. Lima: Universidad Peruano Cayetano Heredia; 2019. (Citado el 8 de junio del 2020) Disponible en: https://www.cayetano.edu.pe/cayetano/ es/estomatologia.

17. Colegio Odontológico del Perú. El COP establece examen de suficiencia profesional a odontólogos extranjeros. Noticop Boletín Informativo del COP. 2015.

18. Sistema Nacional de la Evaluación y Certificación de la Calidad Educativa. Estándares para la Acreditación de la Carrera Profesional Universitaria. Separata 
especial. Lima: Diario El Peruano;12 de julio 2010.

19. Universidad Peruana Cayetano Heredia. Plan curricular de la carrera profesional de Estomatología. Lima: Dirección Universitaria de Gestión de la Docencia, Universidad Peruana Cayetano Heredia; 2016.

20. Sistema Nacional de la Evaluación y Certificación de la Calidad Educativa. Modelo de Acreditación para Programas de Estudios de Educación Superior Universitaria: Explicación de estándares y criterios. Lima: SINEACE; 2017.

21. Nique C. Orientación empática de estudiantes de odontología de una universidad peruana. Kiru. 2013; 10(1): 49-54.

22. Abdelkarim A, Sullivan D. Attitudes and Perceptions of U.S. Dental Student and Faculty Regarding Dental Licensure. 2015; 79(1): 81-88.
23. Del Valle R, Jara E, Del Pino M, et al. Desafíos para la evaluación del perfil de egreso, aproximaciones conceptuales. Temuco, Chile: Universidad Católica de Temuco; 2017.

24. Ruiz C, Manrique J. Acreditación universitaria en las carreras de odontología en el Perú. Rev Estomatol Herediana. 2016; 26(2): 85-91.

Recibido : 08-07-2020

Aceptado : 29-09-2021 